\title{
Randomised Controlled Trial (RCT) of cannabinoid replacement therapy (Nabiximols) for the management of treatment-resistant cannabis dependent patients: a study protocol
}

Anjali K. Bhardwaj ${ }^{1,2,3^{*}}$, David J. Allsop ${ }^{3}$, Jan Copeland ${ }^{4}$, lain S. McGregor ${ }^{3}$, Adrian Dunlop ${ }^{5}$, Marian Shanahan ${ }^{4}$, Raimondo Bruno ${ }^{4,6}$, Nghi Phung $^{7}$, Mark Montebello², Craig Sadler ${ }^{5}$, Jessica Gugusheff ${ }^{1,2}$, Melissa Jackson ${ }^{5}$, Jennifer Luksza', Nicholas Lintzeris ${ }^{1,2}$ and Agonist Replacement for Cannabis Dependence (ARCD) study group

\begin{abstract}
Background: The cannabis extract nabiximols (Sative ${ }^{\circledast}$ ) effectively supresses withdrawal symptoms and cravings in treatment resistant cannabis dependent individuals, who have high relapse rates following conventional withdrawal treatments. This study examines the efficacy, safety and cost-effectiveness of longer-term nabiximols treatment for outpatient cannabis dependent patients who have not responded to previous conventional treatment approaches.

Methods/Design: A phase III multi-site outpatient, randomised, double-blinded, placebo controlled parallel design, comparing a 12-week course of nabiximols to placebo, with follow up at 24 weeks after enrolment.

Four specialist drug and alcohol outpatient clinics in New South Wales, Australia.

One hundred forty-two treatment seeking cannabis dependent adults, with no significant medical, psychiatric or other substance use disorders.

Nabiximols is an oromucosal spray prescribed on a flexible dose regimen to a maximum daily dose of 32 sprays; 8 sprays (total $21.6 \mathrm{mg}$ tetrahydrocannabinol (THC) and $20 \mathrm{mg}$ cannabidiol (CBD)) four times a day, or matching placebo, dispensed weekly. All participants will receive six-sessions of individual cognitive behavioural therapy (CBT) and weekly clinical reviews. Primary endpoints are use of non-prescribed cannabis (self-reported cannabis use days, urine toxicology), safety measures (adverse events and abuse liability), and cost effectiveness (incremental cost effectiveness in achieving additional Quality Adjusted Life Years). Secondary outcomes include, improvement in physical and mental health parameters, substance use other than cannabis, cognitive functioning and patient satisfaction measures.

Discussion: This is the first outpatient community-based randomised controlled study of nabiximols as an agonist replacement medication for treating cannabis dependence, targeting individuals who have not previously responded to conventional treatment approaches. The study and treatment design is modelled upon an earlier study with this population and more generally on other agonist replacement treatments (e.g. nicotine, opioids).
\end{abstract}

Trial registration: Australian and New Zealand Clinical Trial Registry: ACTRN12616000103460 (Registered 1st February 2016).

Keywords: Cannabis, Cannabis withdrawal, Agonist replacement, Nabiximols, Marijuana, Study protocol

\footnotetext{
* Correspondence: anjali.bhardwaj@sydney.edu.au

'Discipline of Addiction Medicine, Central Clinical School, University of

Sydney, Sydney, NSW, Australia

${ }^{2}$ The Langton Centre, Drug and Alcohol Services, South Eastern Sydney Local

Health District, Sydney, NSW, Australia

Full list of author information is available at the end of the article
} 


\section{Background}

Approximately 183 million people aged 15-64 years, (3.8\%) of the global population currently use cannabis worldwide, dwarfing the use of all other globally regulated substances $[1,2]$. In Australia, it is estimated that about 6.6 million people aged 14 years or older have used cannabis at some stage in their lives [3], compared with approximately 22.2 million in the USA $[4,5]$. Close to $10.4 \%$ of adults in Australia between the age of 14 and over have used cannabis in the past 12 months [6], of which approximately $10 \%$ report dependent patterns of use, making it the most common illicit drug of dependence [7].

Cannabis dependence is associated with a range of health problems including cognitive, psychiatric, cardiovascular and respiratory disorders $[1,7,8]$, and considerable societal burden [9]. Cannabis ranks second of all illicit drugs in hospital associated costs [10], is the primary drug of concern in $24 \%$ of Australian alcohol and other drug treatment services (AODTS) [11], and is identified as a problem drug for $55 \%$ of addiction-related treatment episodes [11].

The effectiveness of existing treatments for cannabis use disorders is far from satisfactory but consistent with many other addictive disorders. Reviews of current 'best practice' psychosocial interventions (e.g. cognitive behavioural therapy (CBT)) indicate that around $80 \%$ of patients relapse within 1-6 months [12]. Treatment of acute cannabis withdrawal is associated with similar relapse rates [13, 14]. More effective approaches are required for people seeking treatment for cannabis use related problems, and as with the treatment of other chronic addiction conditions, there is particular interest in the development of treatment approaches that combine medication with psychosocial interventions [15]. Whilst the importance of medication to support current best practice psychosocial interventions has been identified [15], there are as yet no efficacious pharmacotherapies for cannabis dependence [16]. Medication trials for cannabis dependence are an emerging area of research [17]. Most trials have either been laboratory based or focused only on treating withdrawal symptoms during initial abstinence rather than longer term relapse prevention [18]. Medicines tested for withdrawal treatment - with limited efficacy include antidepressants such as bupropion, [19] the mood stabilizers divalproex [20] and lithium [14], the $\alpha_{2}$ _adrenergic agonist lofexidine [21] and the supplement N-acetylcysteine (NAC) [22].

A promising alternative is agonist replacement (or substitution) cannabinoid pharmacotherapies, akin to opioid or nicotine replacement treatment [23]. Studies of cannabinoid agonist medications in withdrawal treatment indicate successful amelioration of withdrawal symptoms. The rationale for agonist medications in cannabis dependence is that they provide a safer route of administration (than smoking), should reduce unsanctioned drug use by preventing withdrawal and reducing cravings [13], and attenuate the acute effects of smoked cannabis [24, 25], potentially facilitating greater engagement in psychosocial interventions. Together, these anticipated effects should provide those also receiving psycho-social interventions and related support to make the necessary lifestyle changes, and distance themselves from drug-related cues, prior to tapering off agonist medication.

Dronabinol, an orally administered synthetic analogue of THC, dose-dependently reduced withdrawal symptoms in the laboratory [20], and improved retention in an outpatient setting [26, 27]. Nabilone, another synthetic THC analogue was efficacious in laboratory experiments, but is as yet untested in clinical settings [21]. A double blind placebo-controlled RCT [13] of nabiximols, an oromucosal spray with approximately equal parts of the cannabinoids THC and CBD, demonstrated successful suppression of withdrawal symptoms and cravings during inpatient detoxification, with greater rates of treatment completion than placebo. However, the high rates of relapse from continuous abstinence following discharge (over two thirds in both groups, but delayed in the nabiximols group) indicate limited longer-term benefits of a 6-day nabiximols regimen.

The high rate of relapse after acute medication-assisted withdrawal highlights the need for longer-term outpatient trials of cannabinoid replacement therapies $[15,16]$.

Only one RCT has examined longer-term cannabinoid agonist replacement treatment. Levin and colleagues [28], compared a 12-week outpatient course of dronabinol to placebo in 121 cannabis dependent treatment seekers. Although dronabinol was well tolerated, had higher treatment retention and reduced withdrawal symptoms, there was no advantage of dronabinol over placebo in achieving abstinence from illicit cannabis, the primary end-point of the study.

The pharmacological profile of nabiximols may have advantages over other available THC agonist medications. Nabiximols is approved in Australia and a number of countries for symptomatic relief of moderate to severe spasticity in multiple sclerosis. It is an oralmucosal spray containing extracts from Cannabis sativa plants grown under licence in the UK by the company GW Pharmaceuticals, containing $2.7 \mathrm{mg} \mathrm{THC}$ and $2.5 \mathrm{mg}$ CBD per $0.1 \mathrm{ml}$ spray, with small amounts $(4 \mathrm{mg} / \mathrm{ml})$ of other plant-derived cannabinoids. The buccal route of administration provides a rapid onset of action and more favourable pharmacokinetics than oral THC or dronabinol [26], more closely mimicking smoked cannabis use. The anxiolytic effects of CBD present in nabiximols may also ameliorate cravings and anxiety associated with dependent cannabis use and withdrawal. 
Two outpatient pilot studies have examined the potential role of nabiximols as an agonist replacement therapy for cannabis withdrawal $[29,30]$. In a withinsubject placebo controlled randomised controlled trial with 8 participants, high nabiximols doses (up to $108 \mathrm{mg}$ THC/100 mg CBD daily) were well tolerated and significantly reduced cannabis withdrawal symptoms in those achieving abstinence from illicit cannabis use [29]. The same research group have recently reported positive clinical findings ( $78 \%$ abstinence) in an open label 12 week course of treatment with nabiximols (average daily dosage $77.5 \mathrm{mgTHC} / 71.7$ mg CBD) and psychological treatment in four participants [30]. These studies highlight nabiximols potential as a longer term agonist replacement approach, however, to date, no outpatient large scale randomised controlled trial has examined the safety and efficacy of nabiximols as a substitution medication in the management of cannabis dependence.

\section{Study objectives}

This study examines the efficacy, safety and costeffectiveness of nabiximols in the outpatient treatment of cannabis dependent treatment seeking patients who have not previously responded to conventional psychosocial interventions. Specific study objectives are:

- Primary Objective 1: To examine the effects of nabiximols vs. placebo on a range of cannabis treatment efficacy outcomes, primarily, changes in illicit cannabis use during treatment and effects on retention in treatment.

- Primary Objective 2: To examine the adverse event profile and abuse liability of nabiximols as a take home treatment for cannabis use disorder.

- Primary Objective 3: To assess the costs and health related quality of life (HRQoL) associated with nabiximols treatment and the potential societal savings (decreased health care, improved productivity, and decreased criminal behaviours).

- Secondary objectives: To examine changes in health related outcomes during outpatient treatment with nabiximols, including a range of mental and physical health dimensions, cognitive function, and psychosocial functioning.

\section{Methods}

\section{Study design}

This project is a phase III multisite (four-sites) outpatient randomised double-blind placebo controlled parallel design comparing a 12-week course of buccally administered nabiximols to placebo (Fig. 1). Both groups receive structured 'best practice' individual counselling based on cognitive behavioural therapy principles, regular case management and clinical reviews over the course of the trial. Medications (nabiximols, placebo) are dispensed on a weekly basis, and study medication is discontinued in week 12 using tapering doses. Participants are followed up at week 24, 12 weeks after discontinuation of nabiximols/placebo treatment. All participants are also followed-up for confidential research interviews, (irrespective of completion of the trial intervention) at baseline (week 0), weeks 4, 8, 12 and 24. Overview of study procedures and sequence of events is provided in Table 1 .

\section{Regulatory and funding}

The design complies with requirements of the Consolidated Standards of Reporting Trials (CONSORT) guideline [31, 32] (see Additional file 1 for SPIRIT CONSORT checklist), and has received ethical clearance from the Human Research Ethics Committee of South East Sydney Local Health District (HREC/14/POWH/701), with relevant local site specific approvals. The University of Sydney is the study sponsor. A National Health and Medical Research Council (NHMRC) of Australia Project Grant (\#1088902) supports the research costs of the study. Health service costs are predominately supported by the participating NSW Health services. Study medications (nabiximols, placebo) are provided free to the study by GW Pharmaceuticals. The study is registered on the Australian New Zealand Clinical Trials Registry (ACTRN12616000103460).

A Data Safety Monitoring Board (DSMB) comprising of an independent statistician, a researcher with cannabinoid expertise, and an addiction medicine specialist with extensive expertise in clinical trials oversees the study.

\section{Sites}

Four clinical sites across three Local Health Districts in New South Wales, Australia were selected to host the study based on their location, history of providing specialist treatment services for cannabis dependent users, and capacity to participate and co-ordinate clinical research activities. Two clinical sites are based in the South East Sydney Local Health District (The Langton Centre and St George Hospital), one in Hunter New England Local Health District (Newcastle Community Health Services) and one in Western Sydney Local Health District (Centre for Addiction Medicine).

\section{Participants \\ Sample size}

Based on the primary outcome measure (efficacy, safety and cost-effectiveness of nabiximols in outpatient treatment of cannabis dependence), a total sample size of 


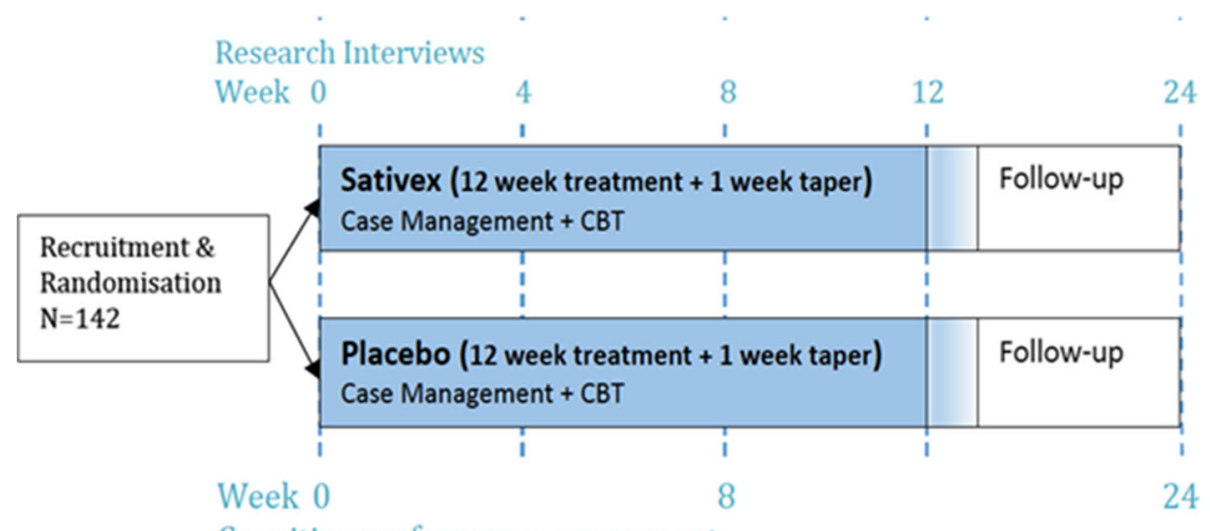

Cognitive performance assessment

Fig. 1 Overview of study design

Table 1 Table of schedule of events

\begin{tabular}{|c|c|c|c|c|c|c|c|c|c|c|c|c|c|c|c|c|}
\hline \multirow[b]{3}{*}{ Timepoint* } & \multicolumn{16}{|c|}{ Study period } \\
\hline & \multirow{2}{*}{$\begin{array}{l}\text { Screen } \\
-t_{1}\end{array}$} & \multirow{2}{*}{$\begin{array}{l}\text { Enrol } \\
0\end{array}$} & \multicolumn{13}{|c|}{ Post-allocation } & \multirow{2}{*}{$\begin{array}{l}\text { Follow up } \\
\text { Wk } 24\end{array}$} \\
\hline & & & $\begin{array}{l}\text { Wk } \\
1\end{array}$ & Wk2 & Wk3 & Wk4 & Wk5 & Wk6 & Wk7 & Wk8 & Wk9 & $\begin{array}{l}\text { Wk } \\
10\end{array}$ & $\begin{array}{l}\text { Wk } \\
11\end{array}$ & $\begin{array}{l}\text { Wk } \\
12\end{array}$ & $\begin{array}{l}\text { Wk } \\
13\end{array}$ & \\
\hline \multicolumn{17}{|l|}{ Enrolment } \\
\hline Phone screen (Eligibility) & $x$ & & & & & & & & & & & & & & & \\
\hline Informed consent for medical screen & $x$ & & & & & & & & & & & & & & & \\
\hline Medical screen/assessment (Eligibility) & $x$ & & & & & & & & & & & & & & & \\
\hline $\begin{array}{l}\text { Informed consent for main study } \\
\text { participation }\end{array}$ & & $x$ & & & & & & & & & & & & & & \\
\hline Allocation & & $x$ & & & & & & & & & & & & & & \\
\hline \multicolumn{17}{|l|}{ Intervention } \\
\hline Medication [Nabiximols or placebo] dispensed & & & $x$ & $x$ & $x$ & $x$ & $x$ & $x$ & $x$ & $x$ & $x$ & $x$ & $x$ & $x$ & $x$ & \\
\hline Nursing clinical reviews & & & $x$ & $x$ & $x$ & $x$ & $x$ & $x$ & $x$ & $x$ & $x$ & $x$ & $x$ & $x$ & $x$ & \\
\hline Medical clinical reviews & & & $x$ & $x$ & & $x$ & & & & $x$ & & & & $x$ & & \\
\hline CBT sessions & & & & $x$ & & $x$ & & $x$ & & $x$ & & $x$ & & $x$ & & \\
\hline Urine drug screen & & $x$ & $x$ & $x$ & $x$ & $x$ & $x$ & $x$ & $x$ & $x$ & $x$ & $x$ & $x$ & $x$ & & $x$ \\
\hline \multicolumn{17}{|l|}{ Assessments } \\
\hline $\begin{array}{l}\text { Research Interviews. Variables include: } \\
\text { Cannabis \& other substance use (TLFB), } \\
\text { CWS, AEs, Aberrant medication behaviour } \\
\text { (mod ORBIT), SF-6D (QOL), WHO Health and } \\
\text { Performance Questionnaire: CT version, } \\
\text { SF-36 (Physical and Mental health), DASS-21, } \\
\text { PHQ-15, OTI: BPI, Crime, Satisfaction, } \\
\text { Test blind (week } 12 \text { only) }\end{array}$ & & $x$ & & & & $x$ & & & & $x$ & & & & $x$ & & $x$ \\
\hline $\begin{array}{l}\text { Clinical (Nursing/medical) Review variables: } \\
\text { AEs, Aberrant medication behaviour (weigh } \\
\text { bottles), ratings dose adequacy, ATOP \& } \\
\text { BPRS at } 4 \text { week intervals, reason for study } \\
\text { termination Wk } 24 \text {. }\end{array}$ & & & $x$ & $x$ & $x$ & $x$ & $x$ & $x$ & $x$ & $x$ & $x$ & $x$ & $x$ & $x$ & & \\
\hline $\begin{array}{l}\text { Cognitive assessment. Variables include } \\
\text { Blood samples (pre/post cognitive testing), } \\
\text { Cognitive testing, Abuse liability (subjective } \\
\text { liking, strength, Physiological response) }\end{array}$ & & $x$ & & & & $x$ & & & & & & & & & & $x$ \\
\hline
\end{tabular}


142 participants (71 per group) is required to achieve around $22 \%$ abstinence rates at 12 -weeks for the placebo group (ascertained by average of previous drug administered clinical trials) and approximately $44 \%$ abstinence rates for the nabiximols group (based on clinical judgement of double the rate of abstinence than placebo) with $80 \%$ power (two-tailed) and $\alpha=0.05$.

\section{Eligibility criteria}

Inclusion criteria are: (a) age 18 to 65 years; (b) meet ICD-10 cannabis dependence criteria; (c) inability to stop cannabis use in previous attempts, operationalised as relapse to cannabis use within one month of a substantive cessation attempt (including prior episodes of counselling, medication, or withdrawal treatment aimed at abstaining from cannabis use), and (d) being willing and able to provide informed consent and follow study procedures, including agreeing to not drive or operate heavy machinery and females of child bearing potential agree to use reliable contraception during the duration of the trial.

\section{Exclusion criteria}

Exclusion criteria for the study are: (a) presence of another substance use disorder other than nicotine or caffeine (i.e. alcohol, other illicit or prescription drug dependence, including methadone or buprenorphine treatment for opioid dependence); (b) severe medical (e.g. severe chronic pain, severe hepatic, cardiovascular or renal impairment) or psychiatric disorder (e.g. unstable schizophrenia, recent drug-induced psychosis, severe mood disorder), assessed at medical screen; (c) women who are pregnant, lactating or planning to become pregnant; (d) concerns regarding safe storage of medication; (e) not available for follow up (e.g. expected travel or incarceration); (f) mandated by court to attend treatment and maintain abstinence for a substance use disorder; (g) history of epilepsy or recurrent seizures; and (i) current active or recent (within past month) treatment for cannabis use disorder.

\section{Early termination criteria}

Early termination criteria for the study include: (i) severe adverse side effects or deteriorating physical or mental health; (ii) violation of treatment centre rules and conditions (e.g. violence towards staff or other patients); (iii) non-compliance with trial protocol, including missing/ not returning medication for more than two consecutive weeks or persistent refusal to participate in trial procedures (such as, non-adherence with study medications, counselling, clinical reviews, case management urine drug screen, bloods, research interviews or monitoring).

\section{Recruitment}

The study is promoted via; (i) referrals from Drug \& Alcohol treatment services; (ii) media advertising, including local newspapers and postcard placements; and (iii) online advertising through University of Sydney website.

A three-step recruitment procedure is used for the study. Interested individuals contact either the central trial coordinator or site-specific researchers by telephone, and are screened for potential eligibility. Information collected at telephone screen relate to the selection criteria, including demographics; recent cannabis and other substance use in past 28 days, Cannabis Severity of Dependence Scale (SDS) score [33]; participation in treatment for substance use disorder in last 28 days; self-reported severe mental and physical health conditions; prior quit attempts; willingness and ability to participate in the study, including weekly clinic attendance. If deemed broadly eligible at telephone screen, the potential participant is invited to attend a detailed medical assessment with a study medical officer at one of the four sites, generally within 1-2 weeks of the telephone screen.

The medical assessment is conducted by a study medical officer (SMO) - an Addiction Medicine Specialist or delegated senior addiction medicine trainee, and involves a comprehensive clinical assessment examining patient goals, substance use and treatment history, physical and mental health, and psychosocial conditions. In addition to the physical and mental state examinations, clinical investigations, such as, urine drug screen for cannabis and other substance use, urine $\beta$-hCG in women to exclude pregnancy, and blood tests are carried out as clinically indicated (e.g. Liver Function Tests if concerned regarding hepatic function). The SMO completes the eligibility checklist following the assessment, and if the patient is eligible and interested in participating in the study, they are referred to the study researcher at each site.

The researcher then provides detailed verbal and written study information, and obtains written informed consent to participate in the study. Upon signing the informed consent form, participants are enrolled into the study.

\section{Randomisation and blinding}

An independent statistician created a randomisation schedule, which was then given to the lead trial pharmacist at each site. Participants are blindly randomised in a 1:1 ratio between groups, using the variable 8-block randomisation schedule of 80 codes per site to maintain blinding. Stratification occurs at each of the four sites (aiming for a 1:1 random allocation at each site). Randomisation and group allocation happen after enrolment (informed consent) and prior to Day 1 of the study, enabling study medications to be prepared by the study pharmacist at each site. The randomisation schedule and 
nabiximols and placebo canisters are only identifiable to trial pharmacists at each site, who themselves have no contact with participants. All participants, clinicians and researchers involved in service delivery, data collection and analysis remain blinded to group allocation (active or placebo) until the end of the study.

\section{Clinical intervention Study medications}

Nabiximols is an oromucosal spray delivered through a mechanically actuated pump, with each spray delivering $100 \mu \mathrm{L}(2.7 \mathrm{mg}$ THC and $2.5 \mathrm{mg}$ CBD). The placebo medication consists of an alcohol base and peppermint oil flavouring present in the active nabiximols medication, but does not include any cannabinoids and plantbased terpenoids. A previous study [13] demonstrated the ability to maintain blind dosing of nabiximols versus placebo in cannabis dependent patients in an inpatient setting. All dispensed medication canisters are labelled with the subjects' name, trial site, week number, medication dose, expiry date and prescribing doctors' name.

The proposed dosing regimen for nabiximols is based upon experience from previous studies with cannabis dependent patients $[13,29,30]$, and the principles of dose titration used in similar clinical paradigms (opioid agonist replacement therapy). Nabiximols and placebo doses are individually titrated by the SMO with each participant to optimise clinical effect (reduce cravings and withdrawal) and safety (avoidance of side effects, such as intoxication). Doses are reviewed and titrated by the clinician and the patient regularly (daily for first two days of Week 1, then at weekly intervals). Day 1 dose is two sprays (5.4 mg THC, $5.0 \mathrm{mg} \mathrm{CBD)} \mathrm{four} \mathrm{times} \mathrm{a} \mathrm{day}$ (QID), days 2 and 3 maximum dose is four sprays $(10.8 \mathrm{mg}$ THC, $10 \mathrm{mg}$ CBD) QID. Thereafter, the maximum daily doses during the 'maintenance' period (to end week 12) are 32 sprays ( $86.4 \mathrm{mg}$ THC, $80 \mathrm{mg}$ CBD) per day divided into four doses (21.6 mg THC, $20 \mathrm{mg}$ CBD QID). These nabiximols doses are considerably greater than those conventionally used for treating other indications (e.g. a daily maximum dose for multiple sclerosis is $32.4 \mathrm{mg}$ THC, $30 \mathrm{mg}$ CBD), reflecting the high THC tolerance of cannabis dependent individuals and the need for high doses to effectively ameliorate withdrawal symptoms and cravings. During week 13, participants have the option of tapered reductions of their study medication at approximately $10-15 \%$ per day, in order to minimise any medication discontinuation effects (e.g. withdrawal).

\section{Clinical reviews}

Weekly clinical reviews with a nurse and/or SMO include: a Time Line Follow Back (TLFB) [34] of their use of study medication, cannabis, nicotine, alcohol and other drugs in the past week since last clinical review; a review of any adverse events; separate participant and clinician assessment of medication dose adequacy (using a 5 -point Likert scale e.g. $1=$ 'much too low', $3=$ 'about right' to $5=$ 'much too high'); participant rating of cannabis withdrawal severity using Cannabis Withdrawal Scale (CWS) [35], general physical and mental health and psychosocial conditions, examination (including blood pressure (BP), pulse rate (PR), evidence of intoxication or withdrawal, or other findings of note) and the opportunity to address other issues identified by the participant. In addition to weekly reviews conducted by nursing staff (baseline, days 1 and 2, weeks 3, 5, 6, 7, 9, 10 , and 11), the SMO reviews the participant at weeks 2 , 4,8 and 12, at which time they also formally assess any adverse events reported since the last medical review and complete the Australian Treatment Outcomes Profile (ATOP) [36], a validated clinician-completed instrument that includes participant ratings of physical and mental health and range of clinical risks, such as, child protection, violence, homelessness) and the Brief Psychiatric Rating Scale (BPRS) [37] to formally assesses psychiatric symptoms that may occur with high dose THC use [38]. The SMO assess whether the participant is globally deteriorating and warrants study discontinuation. See Table 1 for schedule of events.

\section{Psychosocial intervention}

In conjunction with receiving medication treatment, participants are provided with a minimum of six structured counselling sessions based on cognitive behavioural therapy (CBT) [39]. The CBT sessions use a range of strategies and interventions, such as, understanding cannabis and the patient, preparing the patient for change and various strategies, ways to manage withdrawal and relapse prevention. Participation in the counselling program is encouraged, and all participants must attend at least 2 sessions (an initial assessment and one further follow up) in order to continue in the study. Participants may negotiate additional counselling sessions with the therapist beyond the 12-week intervention period. Counsellors complete de-identified research Clinical Record Forms (CRFs) after each session to identify themes or areas covered according to the CBT intervention as a strategy to record adherence with the counselling intervention.

\section{Outcome and treatment process measures}

Outcome measures for each of the study objectives are described below, and summarised in Tables 1 and 2 .

\section{Objective 1}

To examine cannabis treatment efficacy outcomes, including changes in illicit cannabis use during treatment and treatment retention. 


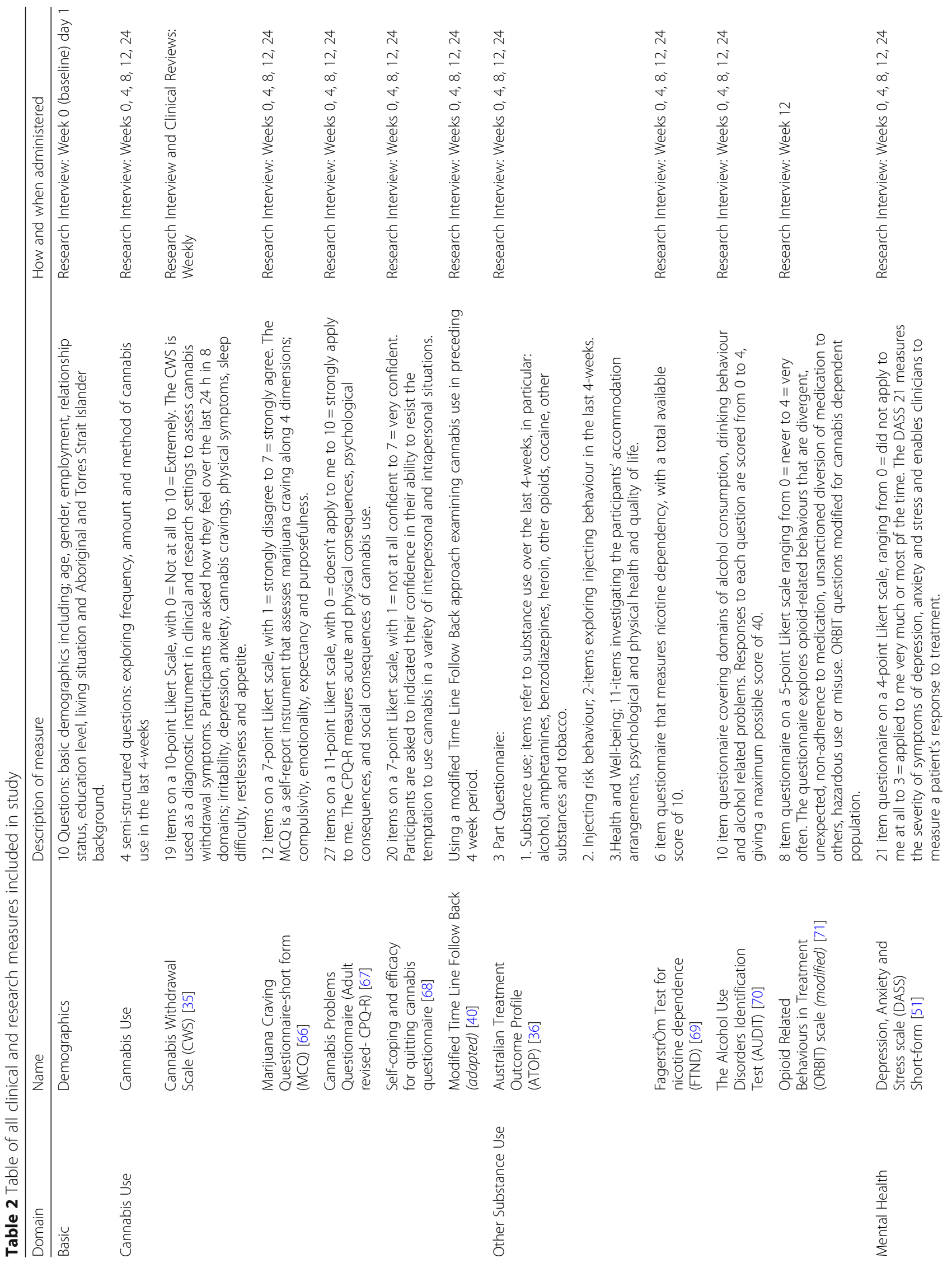




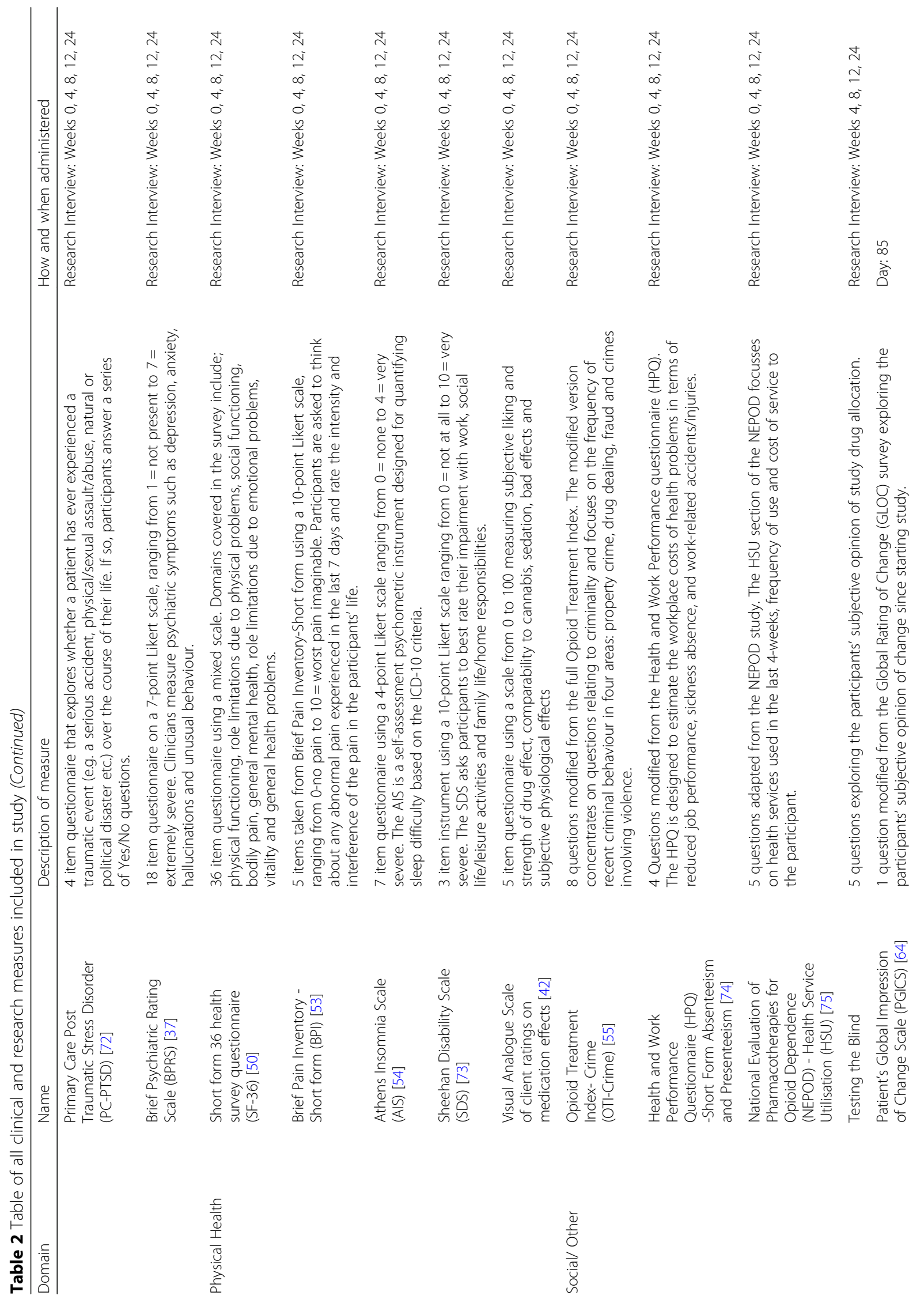




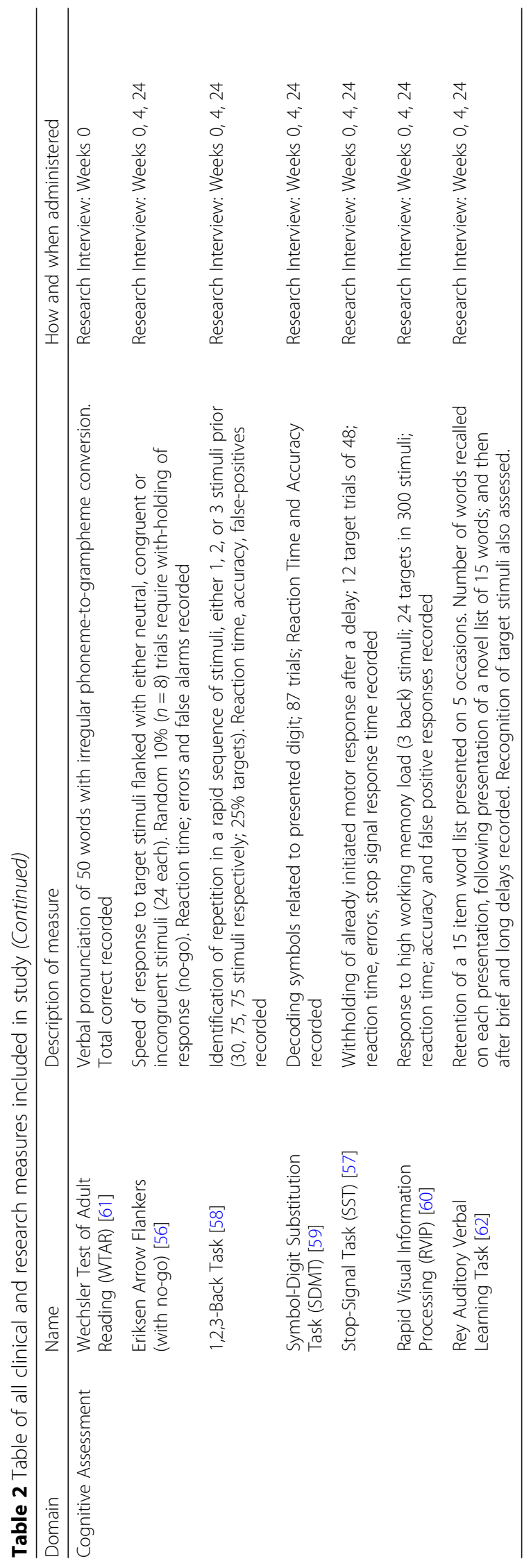


- Illicit cannabis use is quantified as self-reported number of days of illicit cannabis use and average daily amount of cannabis use in grams at the 4 weekly research interviews using modified Timeline Followback [40] techniques. The primary end-point is self-reported illicit cannabis use days during the maintenance phase of treatment (weeks 1-12). Objective measures of illicit cannabis use will be determined from weekly urine collection with quantitative analysis of urinary THC, THC-COOH, $\mathrm{CBD}$ and other cannabinoids. 4-weekly point prevalence abstinence during maintenance phase (weeks $1-4,5-8,9-12$ ), and post-treatment period (week 24) will be ascertained by combining self-report data from researcher interviews and urinalysis.

- Treatment retention (days in protocol treatment) are recorded from clinical records. Participants who do not attend for more than two consecutive weeks are deemed to have dropped out of treatment, and the last scheduled day of dosing is calculated as the end of treatment date.

\section{Objective 2}

To examine the adverse event profile, and the abuse liability of nabiximols as a take home treatment for cannabis use disorder.

- Adverse events are assessed and addressed during clinical assessments with the study medical officer at 4weekly appointments. At the end of study participation, the SMO records the severity of each AE (mild, moderate, severe), the outcome (ongoing or resolved, with or without treatment), and attribution to study medication.

- Abuse liability: Adherence to study medication is estimated by participants returning their medication canisters at the weekly clinical review and weighing the amounts of medication used (equivalent to a pill count). Aberrant medication behaviours (missed doses, extra 'unsanctioned' doses, misuse or diversion) are assessed by self-report at researcher interviews using the modified ORBIT [41], a validated aberrant medication behaviours self-report instrument. In addition, a series of subjective assessments of abuse liability using the Visual Analogue Scale (VAS) [42], (0-100) of subjective liking, comparability to cannabis, strength of effect and subjective physiological effects are conducted 4weekly at researcher interviews.

\section{Objective 3}

To assess the costs and health related quality of life (HRQoL) associated with the provision of nabiximols for treatment of resistant cannabis use disorder and the potential societal savings (decreased health care, improved productivity, and changes in criminal behaviors).

- Cost effectiveness: Consistent with other drug treatment cost effectiveness evaluations [43-45], the primary outcome is Quality Adjusted Life Years (QALY) measured by the SF-6D [46], (at 4 weekly research interviews) determined using area under the curve analysis [47] for each individual. Resources included are all clinical services provided as trial interventions (see Treatment Process Measures below), adverse event management, self-reported health care utilization outside of the trial (hospital, emergency department, primary care and other specialist health services) as well as self-reported participation in criminal activity that will be costed using unit costs (CPI adjusted if necessary) [48]. Lost productivity and personal costs are collected by structured self-report (WHO Health and Performance Questionnaire: Clinical Trials Version) [49]. The costs will be summed and combined with the outcome measure, and the incremental cost-effectiveness ratio $\left[\mathrm{ICER}=\left(\mathrm{C}_{\text {Nabiximols }}-\mathrm{C}_{\text {Control }}\right) /\left(\mathrm{E}_{\text {Nabiximols }}-\mathrm{E}_{\text {Control }}\right)\right]$ calculated.

\section{Secondary objectives}

To examine changes in health-related outcomes during outpatient treatment with nabiximols, including mental and physical health, cognitive performance, and psychosocial functioning

- Other substance use (alcohol, opioids, stimulants, benzodiazepines, tobacco) are recorded by selfreport number of days used in the past 28 days using the ATOP at 4-week research interview.

- Health outcomes and psychosocial function. The SF36 [50] is administered at 4-week research interviews to assess dimensions of physical and mental health and psychosocial function. Mental health will be also be assessed using the Depression, Anxiety and Stress Scale (DASS-21) [51] at 4-weekly research interviews and the BPRS at 4-weekly medical reviews. Patient reported ratings of physical health are also assessed using the Physical Health Questionnaire-15 (PHQ15) [52]. Pain severity and pain interference is assessed using the Brief Pain Inventory [53]. Subjective sleep ratings are assessed using the Insomnia Sleep Inventory [54]. Self-reported drug related crime (e.g. drug dealing, income generating crime) are examined using the Crime Section of the Opiate Treatment Index [55].

- Cognitive function is assessed by the researcher at baseline (week 0 , day 1 ), during the maintenance phase (week 4), and at follow-up (week 24). A 
targeted series of computerised tests sensitive to acute THC effects are conducted, specifically an acute battery: Eriksen Flanker Task [56], Stop Signal Task [57], N-Back [58], Digit-Symbol Substitution [59], and Rapid Visual Information Processing [60] as well as a control measure (Wechsler Test of Adult Reading [61]) and a measure of memory and learning (Ray Auditory Verbal Learning Test, RAVLT [62]). At week 4 assessments, cognitive testing (acute battery) are performed 30 min prior to (trough) and 45-60 min after (peak effects) supervised dosing. Blood samples at trough and peak are taken for plasma cannabinoid levels (THC, 11-OH THC, $\mathrm{THC}-\mathrm{COOH}, \mathrm{CBD}, 11-\mathrm{OH} \mathrm{CBD})$ to assist in the interpretation of findings. The acute battery uses computerised tests within the Penscreen system [63] that create random numbers for stimuli to minimise learning effects. Similarly, repeated memory assessment use parallel versions of the RAVLT. It will be of particular interest to examine whether nabiximols is associated with cognitive improvement relative to Placebo and relative to baseline. Week 24 cognitive performance assessment is examined to investigate within-subject longitudinal changes over time.

- Patient reported outcome and satisfaction measures: Many of the study outcomes use 'patient reported outcome measures' of specific domains. The Patient Global Impression of Change Scale (PGICS) [64] examines the participant's assessment of change in their global condition at week 12 since entering treatment in the trial. Participants are also asked questions relating to their satisfaction of treatment medication and dose and their likelihood of recommending it to others.

\section{Treatment 'process' measures}

details regarding participation in trial interventions are collected on paper clinical record forms (CRF) and include details regarding doses of trial medication used (reported at weekly clinical reviews), participation in medical, counselling and clinical review sessions as well as reasons for study termination (completed protocol, treatment drop-out, medical discharge, administrative discharge, incarceration). At the completion of the 'maintenance' medication phase of the trial (week 12 researcher interview), participants are asked to estimate which medication group they were assigned as a means of testing the blind.

\section{Research procedures}

\section{Research interviews}

The research assistant at each site conducts research interviews at weeks 0 (baseline), 4, 8, 12 and week 24 .
Details of the instruments and timing of administration are shown in Table 2.

\section{Urinalysis and blood pathology testing}

Blood and urine samples are collected in accordance with the National Statement on Ethical Conduct in Human Research (2007). All blood and urine samples are taken and stored de-identified using the patients study ID code.

Urine drug screens are collected during the medical screen and on day 1 of commencing the trial to confirm cannabis use and check for other drug use other than cannabis (prior to study medication being administered). Subsequent urine samples are collected weekly at the clinical review (and at week 24 research interview), and stored at $-20{ }^{\circ} \mathrm{C}$ until the end of the trial, with results not being available to the clinical or research staff until the completion of all data collection. The urine samples will enable cannabinoid metabolite profiles to be charted through time, as a means to identify ongoing illicit cannabis use, using creatinine corrections [65].

Blood samples are taken from all participants at baseline, week 4 (immediately before and after cognitive testing) and week 24 to determine serum cannabinoid (THC, 11-OH THC, THC-COOH, CBD, 7-OH CBD) levels. $10 \mathrm{mls}$ of blood are collected at each time-point and immediately centrifuged at $4{ }^{\circ} \mathrm{C}$ for $15 \mathrm{~min}$ at 1500 RPM and plasma pipetted into $4 \times 1 \mathrm{ml}$ aliquots, immediately frozen at $-20{ }^{\circ} \mathrm{C}$. A Shimadzu 8030 triple quadrupole mass spectrometer will be used to analyse serum cannabinoids (LCMS; Shimadzu Corp, Kyoto, Japan).

\section{Statistical analyses}

Chi square and ANOVA tests will be performed to identify any baseline covariates that differ between groups. Prior to carrying out primary and secondary analysis, the percentage of missing values in the raw dataset and Little's MCAR test will be used to determine if multiple imputation of missing values is required (except for missing urine where cannabis use will be assumed to have taken place). The multiple imputation will be done if the percentage of missing values exceeds $5 \%$.

All analyses will use Intention-to-treat, which is defined as any person who is randomised to one of the study arms and receives at least one dose of study medications. Mixed Models for Repeated Measures (MMRM) will compare groups on changes in outcome variables (cannabis use and secondary outcomes) in the medication phase with the multiply imputed dataset, (assuming that Little's MCAR at Random test confirms the data to be missing at random). In addition, we will perform a sensitivity analysis based on only those with complete data, and compare results to that from the MI dataset analysis. 
Adverse Events will be analysed using chi-square. A Cox proportional hazards model will compare retention in treatment between study arms, controlling for potential confounds. The impact of the intervention on postmedication outcomes will compare changes in cannabis use outcomes at baseline and at follow up between groups using MMRMs.

Family-wise error corrections will control for Type 1 errors where multiple comparisons are performed within a particular analysis where post hoc contrasts are performed to further explore interesting (significant) findings.

\section{Study limitations}

This study has a number of possible limitations. One issue relates to retention in study protocol. Participation in the study is voluntary, and study procedures span 24 weeks, including 13 weeks of study treatment interventions. The lengthy treatment duration in the study is generally longer than conventional treatment of cannabis dependence, and participants may be deterred from entering the trial or prematurely terminate study participation, which in turn may have implications in assessing effectiveness of treatment outcomes and research followup. Similarly, individuals enrolling in a medication study may have different levels of interest or commitment to the CBT in the study, which may enhance heterogeneity of outcomes.

The study uses a flexible dosing approach to medication. Whilst a fixed regimen - or indeed a structured comparison of different doses (e.g. high or low) is often used in many medication studies - experience from opioid agonist clinical and research practice highlights the need to tailor doses to individual requirements, reflecting differing physiological tolerance, adverse event profiles and behavioural characteristics of patients. Nevertheless, the different doses used may increase heterogeneity of outcomes and limit the ability to detect significant differences if there is indeed a major dose effect.

Measures of illicit cannabis use during treatment are the primary study outcome, with self-reported use (via the TLFB) as the primary endpoint. Participants may have a response bias and report inaccurate drug use to both clinicians and researchers. Whilst objective assessment of illicit cannabis use using urine drug screening should enhance self-report validity, it remains to be seen whether illicit cannabis use can be differentiated from prescribed nabiximols use. Various toxicological approaches are being examined to validate the identification of illicit cannabis use in nabiximols prescribed patients (to be described elsewhere).

\section{Conclusion}

There is a need for more effective treatment approaches for cannabis dependent patients who are unable to discontinue their illicit use through psychosocial interventions alone. Longer-term agonist replacement treatment approaches rather than acute withdrawal management are likely to be more effective, with the combination of THC:CBD nabiximols preparation being potentially advantageous over synthetic THC analogues. This is the first large-scale outpatient RCT of nabiximols for this population, and has required the development of clinical and research methods specific to agonist treatment with a plant-derived cannabinoid formulation, building upon clinical research models previously used in opioid agonist treatment approaches.

\section{Additional file}

Additional file 1: CONSORT 2010 checklist of information to include when reporting a randomised trial. (PDF $69 \mathrm{~kb}$ )

\section{Abbreviations}

ATOP: Australian Treatment Outcomes Profile; BPI: Brief Pain Inventory; BPRS: Brief Psychiatric Rating Scale; CBD: Cannabidiol; CBT: Cognitive behavioural therapy; CONSORT: Consolidated Standards of Reporting Trials; CRF: Clinical record form; CWS: Cannabis Withdrawal Scale; DASS21: Depression, Anxiety and Stress Scale-21; DSMB: Data Safety Monitoring Board; HRQoL: Health related quality of life; ISI: Insomnia Sleep Inventory; NHMRC: National Health and Medical Research Council; ORBIT: Opioid Treatment Index- Crime; PGICS: Patient Global Impression of Change Scale; PHQ-15: Physical Health Questionnaire-15; QALY: Quality Adjusted Life Years; QID: Four times a day; RAVLT: Ray Auditory Verbal Learning Test;

RCT: Randomised controlled trial; SF-36: Short form 36 health survey questionnaire; SMO: Study medical officer; THC: Tetrahydrocannabinol; TLFB: Timeline Follow Back; VAS: Visual Analogue Scale

\section{Acknowledgements}

Agonist Replacement for Cannabis Dependence (ARCD) study group: Drug and Alcohol Services, South Eastern Sydney Local Health District, Senior Trial Pharmacist: Therese Chan

Drug and Alcohol Services, South Eastern Sydney Local Health District key staff: Raelene Dojcinovic, Betty Jago, Lynsey McKendrick, Consuelo Rivas, Ricardo Schwanz, Abigail Yang, Zachary Zavareh.

Drug and Alcohol Clinical Services, Hunter New England Local Health District key staff: Michelle Hall, Susan Hazelwood, Anthony Winmill.

Centre for Addiction Medicine, Western Sydney Local Health District key staff: Angelo Barbaro, Kerin Black, Tim Ho, Meryem Jefferies, Jonathon Coreas Jule, Shyam Nagubandi, Mahsa Shahidi, Catherine Silsbury Lisa Snell, Matthew Wijanto.

\section{Funding}

This study was funded by National Health and Medical Research Council, project grant \#1088902. Study drug (nabiximols and placebo) were provided free of charge by GW Pharmaceuticals, UK.

\section{Availability of data and materials}

Not applicable.

\section{Authors' contributions}

The study design was conceived of and designed by NL, DA, JC, IM, AD, MS, $R B, N P, M M, C S$. $A B$ contributed to revisions in the study design, overall coordination of all sites and drafting the manuscript. JG, MJ and JL contributed in revisions to the manuscript and site-specific coordination. All authors have read and approved the final manuscript. 
Local Health District received site-specific ethical approval. All participants signed a Participant Information Consent Form [PICF] before being enrolled in the study. Informed consent involved a 3-step process. 1. Verbal information about the trial was given to potential participant either face-toface or over the telephone during an initial screen. 2. Participants eligible for a medical screen were asked to sign a 'consent to participate in a medical screen' form before seeing the trial medical officer. 3. If participant was deemed eligible by trial medical officer, the medical officer/trial researcher verbally discussed the trial to the participant and invited any queries or concerns to be addressed. The participant was given a copy of the main PICF to take home and review. The PICF was signed by the participant and a witness on day 1 of commencing the trial.

\section{Consent for publication}

The participant information consent form includes a section relating to consent for de-identified data to be published. This is however not applicable for this paper.

\section{Competing interests}

The authors declare that they have no competing interests.

\section{Publisher's Note}

Springer Nature remains neutral with regard to jurisdictional claims in published maps and institutional affiliations.

\section{Author details \\ ${ }^{1}$ Discipline of Addiction Medicine, Central Clinical School, University of Sydney, Sydney, NSW, Australia. ${ }^{2}$ The Langton Centre, Drug and Alcohol Services, South Eastern Sydney Local Health District, Sydney, NSW, Australia. ${ }^{3}$ School of Psychology, University of Sydney, Sydney, NSW, Australia. ${ }^{4}$ National Drug and Alcohol Research Centre, University of New South Wales, Sydney, NSW, Australia. ${ }^{5}$ Drug and Alcohol Clinical Services, Hunter New England Local Health District, Newcastle, NSW, Australia. ${ }^{6}$ School of Medicine, University of Tasmania, Hobart, TAS, Australia. ${ }^{7}$ Centre for Addiction Medicine, Cumberland Hospital, Western Sydney Local Health District, Sydney, NSW, Australia.}

\section{Received: 31 August 2017 Accepted: 28 March 2018} Published online: 18 May 2018

\section{References}

1. UNODC. World Drug Report 2013. 2013: United Nations Publication, Sales No. E.13.XI.6.

2. UNODC. World drug report 2015. Vienna: United Nations Office on Drugs and Crime; 2015.

3. AlHW. Alcohol and other drug treatment services in Australia 2011-12. Canberra: AlHW; 2013

4. Hasin DS, et al. Prevalence of marijuana use disorders in the United States between 2001-2002 and 2012-2013. JAMA psychiatry. 2015;72(12):1235-42.

5. Quality, C.f.B.H.S.a., 2015 National Survey on Drug Use and Health: Detailed Tables., in Substance Abuse and Mental Health Services Administration. 2016: Rockville, Maryland.

6. AlHW. In: D. Statistics, editor. 2016 National Drug Strategy Household Survey: preliminary findings. Canberra: AlHW; 2017

7. Hall W, Degenhardt L. Adverse health effects of non-medical cannabis use Lancet. 2009:374(9698):1383-91.

8. WHO. The health and social effects of nonmedical cannabis use. Geneva: Department of Mental Health and Substance Abuse (MSD); 2016. p. 95.

9. Roffman R, Stephens R. In: Edwards PG, editor. Cannabis dependence: its nature, consequences and treatment. International research monographs in the addictions. Cambridge: Cambridge University Press; 2006.

10. Collins D, Lapsley H. The costs of tobacco, alcohol and illicit drug abuse to Australian society in 2004-05. Canberra: D.o.H.a. Ageing; 2008.

11. AlHW. Alcohol and other drug treatment services in Australia 2014-2015. In: Drug and treatment series no.27. Canberra: AlHW; 2016.

12. Gates, P.J., et al., Psychosocial interventions for cannabis use disorder. Cochrane Database Syst Rev, 2016(5).

13. Allsop DJ, et al. Nabiximols as an agonist replacement therapy during Cannabis withdrawal: a randomized clinical trial. JAMA Psychiatry. 2014; 71(3):281-91.
14. Johnston J, et al. A double blind, randomised, placebo controlled trial of lithium carbonate for the management of cannabis withdrawal. In: Drug and alcohol review. Hoboken: Wiley; 2013.

15. Danovitch I, Gorelick DA. State of the art treatments for cannabis dependence. Psychiatr Clin N Am. 2012;35(2):309-26.

16. Vandrey R, Haney M. Pharmacotherapy for cannabis dependence: how close are we? CNS Drugs. 2009;23(7):543-53.

17. Copeland J, Clement N, Swift W. Cannabis use, harms and the management of cannabis use disorder. Neuropsychiatry. 2014;4(1):55-63.

18. Marshall K, et al. Pharmacotherapies for cannabis dependence. Cochrane Database Syst Rev. 2014;12:Cd008940.

19. Carpenter KM, et al. A preliminary trial: double-blind comparison of nefazodone, bupropion-SR, and placebo in the treatment of cannabis dependence. Am J Addict. 2009;18(1):53-64.

20. Haney $\mathrm{M}$, et al. Marijuana withdrawal in humans: effects of oral THC or divalproex. Neuropsychopharmacology. 2004;29(1):158-70.

21. Haney $\mathrm{M}$, et al. Effects of THC and lofexidine in a human laboratory model of marijuana withdrawal and relapse. Psychopharmacology. 2008;197(1): 157-68.

22. Gray KM, et al. A double-blind randomized controlled trial of $\mathrm{N}$ acetylcysteine in cannabis-dependent adolescents. Am J Psychiatr. 2012; 169(8):805-12.

23. Darke S, Farrell M. Would legalizing illicit opioids reduce overdose fatalities? Implications from a natural experiment. Addiction. 2014;109(8):5.

24. Budney AJ, et al. Adults seeking treatment for marijuana dependence: a comparison with cocaine-dependent treatment seekers. Exp Clin Psychopharmacol. 1998;6(4):419-26.

25. Karst M, Wippermann S, Ahrens J. Role of cannabinoids in the treatment of pain and (painful) spasticity. Drugs. 2010;70(18):2409-38.

26. Karschner EL, et al. Plasma cannabinoid pharmacokinetics following controlled oral delta9-tetrahydrocannabinol and oromucosal cannabis extract administration. Clin Chem. 2011:57(1):66-75.

27. Huestis MA, et al. Characterization of the absorption phase of marijuana smoking. Clin Pharmacol Ther. 1992:52(1):31-41.

28. Levin FR, et al. Dronabinol for the treatment of cannabis dependence: a randomized, double-blind, placebo-controlled trial. Drug Alcohol Depend. 2011;116(1-3):142-50.

29. Trigo JM, et al. Effects of fixed or self-titrated dosages of Sativex on cannabis withdrawal and cravings. Drug Alcohol Depend. 2016;161:298-306

30. Trigo JM, et al. Sativex associated with behavioral-relapse prevention strategy as treatment for Cannabis dependence: a case series. J Addict Med. 2016:10(4):274-9.

31. Schulz KF, et al. CONSORT 2010 statement: updated guidelines for reporting parallel group randomised trials. BMJ. 2010;340:c332.

32. Chan A-W, et al. SPIRIT 2013 statement: defining standard protocol items for clinical trials. Ann Intern Med. 2013;158(3):200-7.

33. Martin G, et al. The severity of dependence scale (SDS) in an adolescent population of cannabis users: reliability, validity and diagnostic cut-off. Drug Alcohol Depend. 2006;83(1):90-3

34. Sobell, L.C. and M.B. Sobell, Timeline follow-back, in Measuring alcohol consumption. 1992, Springer. p. 41-72.

35. Allsop, D.J., M. Norberg, and J. Copeland, Development of a cannabis withdrawal scale. Centrelines, 2010.

36. Ryan $\mathrm{A}$, et al. Validation and implementation of the Australian treatment outcomes profile in specialist drug and alcohol settings. Drug Alcohol Rev 2014;33(1):33-42.

37. Overall JE, Gorman DR. The brief psychiatric rating scale. Psychol Rep. 1962; 10:22.

38. D'Souza DC, et al. The psychotomimetic effects of intravenous delta-9tetrahydrocannabinol in healthy individuals: implications for psychosis. Neuropsychopharmacology. 2004;29(8):1558-72.

39. Copeland J. Marijuana brief intervention: an SBIRT approach. Center City: Hazelden Publishing; 2017

40. Norberg MM, Mackenzie J, Copeland J. Quantifying cannabis use with the timeline Followback approach: a psychometric evaluation. Drug Alcohol Depend. 2012;121(3):247-52

41. Mattick, R.P. et al. Minimizing the unintended consequences of opioid treatment: development of a drug behavior scale for use in Australia. In College on Problems of Drug Dependence, 2012. Palm Spings, USA

42. Crichton N. Visual analogue scale (VAS). J Clin Nurs. 2001;10(5):706. 
43. Connock, M., et al., Methadone and buprenorphine for the management of opioid dependence: a systematic review and economic evaluation. 2007.

44. Doran CM, et al. Buprenorphine versus methadone maintenance: a costeffectiveness analysis. Drug Alcohol Depend. 2003;71(3):295-302.

45. Harris AH, Gospodarevskaya E, Ritter AJ. A randomised trial of the cost effectiveness of buprenorphine as an alternative to methadone maintenance treatment for heroin dependence in a primary care setting. PharmacoEconomics. 2005;23(1):77-91.

46. Brazier JE, Roberts J. The estimation of a preference-based measure of health from the SF-12. Med Care. 2004;42(9):851-9.

47. Drummond C, et al. Effectiveness and cost-effectiveness of a stepped care intervention for alcohol use disorders in primary care: pilot study. $\mathrm{Br} J$ Psychiatry. 2009;195(5):448-56.

48. Rolling K. Counting the costs of crime in Australia: a 2005 update. Canberra: A.l.o. Criminology; 2008.

49. Organisation, W.H., World Health Organization health and performance questionnaire (HPQ): clinical trials baseline version. 2002.

50. Ware JE Jr, Sherbourne CD. The MOS 36-item short-form health survey (SF-36). I. Conceptual framework and item selection. Med Care. 1992;30(6):473-83.

51. Lovibond SH, Lovibond PF. Manual for the depression anxiety stress scales. Sydney: Psychology Foundation; 1995.

52. Kroenke K, Spitzer RL, Williams JB. The PHQ-15: validity of a new measure for evaluating the severity of somatic symptoms. Psychosom Med. 2002; 64(2):258-66.

53. Cleeland C, Ryan K. Pain assessment: global use of the brief pain inventory. Ann Acad Med Singap. 1994;23(2):129-38.

54. Soldatos CR, Dikeos DG, Paparrigopoulos TJ. Athens insomnia scale: validation of an instrument based on ICD-10 criteria. J Psychosom Res. 2000; 48(6):555-60.

55. Darke $\mathrm{S}$, et al. Development and validation of a multi-dimensional instrument for assessing outcome of treatment among opiate users: the opiate treatment index. Br J Addict. 1992:87(5):733-42.

56. Eriksen B, Eriksen C. Effects of noise letters upon the identification of a target letter in a nonsearch task. Percept Psychophys. 1974;16(1):143-9.

57. Logan GD, Schachar RJ, Tannock R. Impulsivity and inhibitory control. Psychol Sci. 1997;8:60-4.

58. Jonides J, et al. Verbal working memory load affects regional brain activation as measured by PET. J Cogn Neurosci. 1997;9(4):462-75.

59. Mattila M, Mattila-Evenden M. Effects of alcohol and hypnosedative drugs on digit-symbol substitution: comparison of two different computerized tests. J Psychopharmacol. 1997;11(4):313-7.

60. Wesnes K, Warburton DM. Effects of smoking on rapid information processing performance. Neuropsychobiology. 1983;9(4):223-9.

61. Wechsler, D. Wechsler Test of Adult Reading: WTAR. 2001: Psychological Corporation

62. Rey, A., L'examen psychologique dans les cas d'encéphalopathie traumatique.(Les problems.). 1941. Archives de psychologie.

63. Cameron E, Sinclair W, Tiplady B. Validity and sensitivity of a pen computer battery of performance tests. J Psychopharmacol. 2001;15(2):105-10.

64. Hurst $\mathrm{H}$, Bolton J. Assessing the clinical significance of change scores recorded on subjective outcome measures. J Manip Physiol Ther. 2004; 27(1):26-35.

65. Huestis MA, Mitchell JM, Cone EJ. Urinary excretion profiles of 11-Nor-9Carboxy- $\Delta$-tetrahydrocannabinol in humans after single smoked doses of marijuana. J Anal Toxicol. 1996;20(6):441-52.

66. Heishman SJ, Singleton EG, Liguori A. Marijuana craving questionnaire: development and initial validation of a self-report instrument. Addiction. 2001;96(7):1023-34.

67. Copeland J, et al. The Cannabis problems Questionair: factor structure reliability, and validity. Drug Alcohol Depend. 2005;80:313-9.

68. Stephens RS, Wertz JS, Roffman RA. Self-efficacy and marijuana cessation: a construct validity analysis. J Consult Clin Psychol. 1995;63(6):1022-31.

69. Heatherton TF, et al. The Fagerstrom test for nicotine dependence: a revision of the Fagerstrom tolerance questionnaire. Br J Addict. 1991;86(9):1119-27.

70. Saunders JB, et al. Development of the alcohol use disorders screening test (AUDIT). WHO collaborative project on early detection of persons with harmful alcohol consumption. Addiction. 1993;88:791-804.

71. Larance $B$, et al. Development of a brief tool for monitoring aberrant behaviours among patients receiving long-term opioid therapy: the opioidrelated Behaviours in treatment (ORBIT) scale. Drug Alcohol Depend. 2016; 159:42-52.
72. Cameron RP, Gusman D. The primary care PTSD screen (PC-PTSD): development and operating characteristics. Primary Care Psychiatry. 2003; 9(1):9-14.

73. Leon $\mathrm{AC}$, et al. Assessing psychiatric impairment in primary care with the Sheehan disability scale. Int J Psychiatry Med. 1997;27(2):93-105.

74. Kessler RC, et al. The world health organization health and work performance questionnaire (HPQ). J Occup Environ Med. 2003;45(2):156-74.

75. Shanahan MD, et al. A cost-effectiveness analysis of heroin detoxification methods in the Australian National Evaluation of pharmacotherapies for opioid dependence (NEPOD). Addict Behav. 2006;31(3):371-87.

\section{Submit your next manuscript to BioMed Central and we will help you at every step:}

- We accept pre-submission inquiries

- Our selector tool helps you to find the most relevant journal

- We provide round the clock customer support

- Convenient online submission

- Thorough peer review

- Inclusion in PubMed and all major indexing services

- Maximum visibility for your research

Submit your manuscript at www.biomedcentral.com/submit
Biomed Central 